\title{
Infarto do miocárdio recorrente sob a perspectiva do familiar da vítima: relato de caso
}

\author{
Recurrent myocardial infarction from the perspective of the victim's family \\ member: case report
}

Taynara Maria dos Santos Dias¹, Suellen Rodrigues de Oliveira Maier¹, Joaquim Rosa Soares Júnior', Gelson Aguiar da Silva Moser², Bruno da Silva Santos ${ }^{3}$, Wanmar de Souza Oliveira ${ }^{1}$

${ }^{1}$ Universidade Federal de Mato Grosso (UFMT) - Rondonópolis (MT), Brasil.

${ }^{2}$ Universidade Federal da Fronteira Sul (UFFS) - Chapecó (MT), Brasil.

Universidade Federal de Mato Grosso (UFMT) - Cuiabá (MT), Brasil.

DOI: https://dx.doi.org/10.7322/abcshs.v44i3.1205

\section{RESUMO}

Introdução: Este estudo relata possíveis fatores para a recorrência de infarto agudo do miocárdio de um paciente atendido em unidade coronariana sob a perspectiva familiar. Relato de Caso: Trata-se de um estudo descritivo, com abordagem qualitativa, do tipo relato de caso. Os dados foram coletados através de uma entrevista piloto semiestruturada, realizada com um familiar de uma paciente que se encontrava internada na unidade coronariana com recorrência de Infarto Agudo do Miocárdio (IAM). O familiar relatou que a intensa atividade física realizada pela paciente, o consumo excessivo de álcool e a não adesão ao tratamento medicamentoso contribuíram para o segundo quadro de IAM. A familiar mencionou que as orientações recebidas durante a alta foram somente em torno dos medicamentos prescritos, destacando a ausência de orientações acerca das alterações no estilo de vida, em especial à equipe de enfermagem. Conclusão: o presente relato revelou que as orientações durante a alta não estão sendo efetivas na prevenção da recorrência de IAM e ressalta a necessidade de repensar o papel dos profissionais de enfermagem em relação às orientações prestadas, como forma de facilitar a adesão do paciente ao tratamento medicamentoso e às mudanças no estilo de vida.

Palavras-chave: infarto do miocárdio; relatos de casos; família; relações familiares; promoção da saúde; prevenção de doenças.

\begin{abstract}
Introduction: This study reports the possible factors for the recurrence of acute myocardial infarction of a patient attended in the coronary care unit in the perspective of relatives. Case Report: This is a descriptive study, with qualitative approach, of the case report type. The data were collected through a semi-structured pilot interview, conducted with a member of the patient family, who was hospitalized in the coronary care unit with the recurrence of Acute Myocardial Infarction (AMI). The relative reported that the intense physical activity performed by the patient, the excessive consumption of alcohol and non-adherence to drug therapy, have contributed to the second event of AMI. The same mentioned that the guidance received during the high, were only around for medicines prescribed, highlighting the absence of guidance about the changes in life style and the realization of the same by health care professionals, in particular nursing staff. Conclusion: The present report revealed that the guidelines during the high are not being effective in the prevention of the recurrence of AMI, and highlights the need to rethink the role of professional nursing in relation to the guidelines provided, as a way of facilitating the patient's adherence to medication treatment and changes in lifestyle.
\end{abstract}

Keywords: myocardial infarction; case reports; family; family relations; health promotion; disease prevention.

Recebido em: 14/09/2018

Revisado em: 28/04/2019

Aprovado em: 09/05/2019

Autor para correspondência: Suellen Rodrigues de Oliveira Maier - Universidade Federal de Mato Grosso - Avenida dos Estudantes $\mathrm{n}^{\circ} 5055$ - Cidade Universitária - CEP: 78735-910 - Rondonópolis (MT), Brasil - E-mail: suellenenf@ufmt.br

Conflito de interesses: nada a declarar. 


\section{INTRODUÇÃO}

A doença cardiovascular (DCV) é considerada uma das predominantes causas de mortalidade e morbidade no mundo, sendo responsável, nas últimas décadas, por 17 milhões de mortes a nível global ${ }^{1}$. No Brasil, cerca de $30 \%$ dos casos de óbito é resultante de doenças circulatórias, sendo a cardiopatia isquêmica uma das responsáveis por $53,8 \%$ das mortes para cada 100 mil pessoas ${ }^{2}$. Além da mortalidade, estas doenças podem causar limitações físicas que influenciam diretamente a qualidade de vida ${ }^{3}$.

A DCV é condição que abrange o sistema circulatório, em que se compreende o coração e os vasos sanguíneos. Caracteriza-se por problemas que afetam as artérias que fornecem sangue ao músculo cardíaco ${ }^{4}$.

A Síndrome Coronariana Aguda (SCA) engloba a Angina instável (AI) e o infarto agudo do miocárdio (IAM) com ou sem Supradesnivelamento do Segmento ST. É um termo que se utiliza para retratar um conjunto de diagnósticos clínicos ocasionado pela obstrução das artérias coronárias devido à instabilidade entre o suprimento e a quantidade necessária de oxigênio no músculo cardíaco $^{5}$.

Dentre as doenças do aparelho circulatório destaca-se o IAM, que se caracteriza como um processo em que áreas de células miocárdicas são destruídas de maneira permanente em virtude de um fluxo sanguíneo reduzido em uma artéria coronária. As manifestações clínicas mais comuns são: precordialgia violenta e constritiva, que pode irradiar para os ombros e os braços, acompanhada de dispneia aliada ou não à taquipneia, palidez cutânea, sudorese, confusão mental, náusea e vômitos ${ }^{6}$.

As doenças cardiovasculares se desenvolvem devido à exposição, ao longo da vida, a diversos fatores de risco, dentre os quais podem ser classificados como: causas não modificáveis, que incluem idade, sexo e antecedentes familiares, e os fatores modificáveis como tabagismo, alimentação inadequada, obesidade, sedentarismo e uso excessivo de álcool. Diante do exposto, percebe-se que medidas simples, como a alteração no estilo de vida, podem diminuir os riscos de desenvolver doenças cardiovasculares? ${ }^{7}$.

As pessoas que vivenciaram um quadro de IAM obrigatoriamente necessitam modicar seus hábitos de vida, com alterações no seu cotidiano. Todas essas ações repercutem em sua vida, gerando mudanças abruptas que, quando não acatadas, levam à recidiva motivada pela não aderência ao tratamento, composto por mudanças nos hábitos alimentares e no tabagismo, práticas regulares de atividades físicas e adesão ao tratamento medicamentoso. Essas mudanças irão resultar em alterações no estilo de vida da pessoa $^{8}$. Além disso, é imperativo enfatizar o papel dos familiares, uma vez que podem colaborar positivamente no que se refere à adesão às medidas preventivas, com vistas a evitar novos eventos.

Neste contexto, diante da questão de pesquisa: "Quais os possíveis fatores favoráveis ao desenvolvimento de um infarto agudo do miocárdio recorrente sob a perspectiva do familiar?".
Objetivou-se relatar a recorrência do infarto agudo do miocárdio de um paciente atendido em unidade coronariana, sob a perspectiva familiar.

\section{RELATO DE CASO}

Trata-se de um estudo descritivo com abordagem qualitativa do tipo relato de caso a partir de uma entrevista piloto com o familiar de um paciente internado na unidade de terapia intensiva coronariana. $\mathrm{O}$ presente manuscrito foi elaborado de modo a contemplar os dados oriundos do projeto de pesquisa matriz: O (re)infarto agudo do miocárdio: concepções e atitudes frente à disfunção cárdica, realizado com pacientes que sofreram recorrência de IAM, bem como com seus respectivos familiares, após a apreciação ética e emissão do parecer consubstanciado aprovado, sob o n. ${ }^{\circ} 1.931 .153$ e certificação de apreciação ética CAAE: 62895316.8.0000.8088.

O presente relato de caso foi estruturado a partir de uma entrevista de conversação, em consonância com a pesquisa convergente assistencial ${ }^{9}$ com um familiar de uma paciente que se encontrava internada na unidade coronariana de um hospital de referência, localizado ao sul do estado de Mato Grosso, Brasil, devido a um IAM recorrente.

A entrevista piloto ocorreu no ambiente hospitalar durante a internação do paciente. Na ocasião, o familiar estava acompanhando a paciente em questão após a alta da unidade intensiva para a unidade não intensiva (enfermaria). Para a organização dos dados do estudo utilizou como ferramenta o instrumento Case Report Guidelines (CARE), com o objetivo de nortear a elaboração do presente relato de caso $^{10}$ no que concerne aos aspectos estruturais do manuscrito.

A análise da entrevista ocorreu após a transcrição da conversação na íntegra e leitura para a extração das unidades de significado, seguindo os preceitos da análise temática ${ }^{11}$ acerca das atividades diárias do paciente entre o primeiro e o segundo evento isquêmico, expressadas pelas unidades de textos selecionadas para compor a descrição do caso na perspectiva do familiar.

O IAM é uma doença que afeta significativamente a vida de uma pessoa, acarretando em uma serie de alterações em seu estilo de vida, como mudanças nos seus hábitos alimentares, atividades físicas e necessidade de tratamento medicamentoso. A família possui um papel fundamental nesse processo de mudança, uma vez que auxiliam a pessoa doente oferecendo apoio durante o tratamento e estando presente nos momentos difíceis.

De modo a compreender quais motivos que provavelmente levaram à recorrência do IAM, o familiar discorreu sobre as atividades diárias da mãe, enfatizando situações acerca do estilo de vida contemplando o aspecto laboral, pessoal e de lazer. 
A paciente trata-se de uma idosa do sexo feminino, de 66 anos de idade, que possui grau de escolaridade incompleto, atualmente é aposentada e não executa nenhum tipo de atividade profissional. $\mathrm{O}$ familiar não residia com a paciente, realizando visitas diárias à mãe.

Ao longo da entrevista, a filha expôs que a mãe havia sofrido o primeiro quadro de infarto há cinco anos, em que foi submetida a cateterismo e angioplastia, posterior à internação na unidade coronariana, até receber alta da instituição hospitalar.

De acordo com a descrição do familiar, o novo evento ocorreu motivado pelas intensas atividades físicas decorrentes do trabalho no campo, o consumo excessivo de bebida alcoólica e à não adesão ao tratamento medicamentoso. A mesma mencionou que a idosa referia episódios de dores persistentes, consideradas leve pela própria paciente, que não procurava a unidade de saúde, apesar do ente famíliar insistir em levá-la ao centro de saúde mais próximo de sua residência.

"Eu acho que foi fato dela fazer muito serviço pesado, devido também da bebida e devido dela não tomar os medicamentos [...] o trabalho no sol quente, trabalhar muito no pesado, pegar peso, e os medicamentos que ela tomou dois meses só, após a angioplastia que ela fez, e o álcool também”. (familiar)

Quando questionada a respeito das orientações que havia recebido pelos profissionais da saúde durante a alta hospitalar quanto à necessidade de alterações nos hábitos de vida da idosa, no tocante aos fatores de risco modificáveis e à adesão ao tratamento medicamentoso, a mesma relatou que havia recebido informações somente em relação aos medicamentos prescritos.

"O doutor explicou que ela tinha que tomar os medicamentos enquanto vida ela tivesse, que ela não podia trabalhar no sol, não podia fazer serviços pesados como ela fazia antes [...]”. (familiar)

Vale ressaltar que a idosa não seguiu corretamente o tratamento medicamentoso, uma vez que utilizou os medicamentos apenas dois meses após retornar para o domicílio. No decorrer do relato, o familiar alega que as únicas orientações que havia recebido no momento da alta foram realizadas pelo médico em relação ao tratamento medicamentoso. Desta forma, verifica-se o déficit em relação às informações relacionadas aos fatores de risco modificáveis como tabagismo, alimentação inadequada, obesidade, sedentarismo, uso excessivo de álcool, fatores que são responsáveis pelo aumento da taxa de recorrência de IAM. E, além disso, destaca-se a ausência de orientações pelos demais profissionais da equipe de saúde.

\section{DISCUSSÃO}

A partir desse estudo, com base no relato realizado pelo familiar da paciente, foi possível inferir que há déficit no que concerne às orientações acerca dos cuidados relacionados ao caráter preventivo das doenças cardiovasculares, em especial os cuidados relacionados a outros eventos obstrutivos no miocárdio.

As orientações no momento da alta hospitalar não são efetivas, o que não contribui no sentido de evitar a recorrência do IAM, visto que os cuidados em saúde, após a alta hospitalar, não foram eficientes devido à falta de orientação, o que favoreceu o surgimento de um novo infarto do miocárdio.

Portanto, faz-se necessário enfatizar a importância da equipe multiprofissional neste processo, em especial o papel da enfermagem, no que concerne em ações e orientações ofertadas durante o processo de hospitalização até o momento da alta hospitalar de um paciente com evento de IAM. Realizando, ainda no contexto hospitalar, implementações de programas educativos específicos e permanentes para esta linha de cuidado, destacando ações de enfermagem na produção de medidas de prevenção e reabilitação cardiovascular (RCV), assegurando condição físicas, mentais e sociais, com enfoque na gravidade da doença e mudanças nos hábitos de vida.

Assim como ofertar ao paciente e aos familiares informações relevantes acerca do contexto de saúde, a fim de evitar novas internações em virtude de complicações inerentes a cuidados inadequados em domicílio, enfatizando a importância da adesão ao tratamento medicamentoso, acompanhamento periódico dos profissionais de saúde no âmbito da atenção primária e mudança no estilo de vida referente aos fatores de risco modificáveis do paciente.

\section{REFERÊNCIAS}

1. Simão AF, Précoma DB, Andrade JP, Correa Filho $H$, Saraiva JFK, Oliveira GMM, et al. I Diretriz Brasileira de Prevenção Cardiovascular. Arq Bras Cardiol. 2013:101(6 Supl. 2):1-63. http://dx.doi.org/10.5935/abc.2013S012

2. Mont'Alverne Filho JR, Lemos Neto PA. Efeitos dos novos antiagregantes plaquetários prasugrel e ticagrelor, administrados up stream, sobre os achados angiográicos da angioplastia primária. Tese (Doutorado) - Universidade de São Paulo. São Paulo: 2015; p. 60-102

3. Teston EF, Cecilio HPM, Santos AL, Arruda GO, Radovanovic CAT, Marcon SS. Fatores associados às doenças cardiovasculares em adultos. Rev Medicina. 2016;49(2):95-102. https://doi.org/10.11606/issn.2176-7262.v49i2p95-102 
4. Aehlert B. Síndromes Coronarianas Agudas. In: ACLS: Suporte avançado de vida em cardiologia: emergência em cardiologia. 4 ed. Rio de Janeiro: Elsevier, 2013; p. 211-75.

5. Almeida MS, Carvalho EMF, Montenegro SML, Silva OB. Síndrome coronariana aguda: diferenças epidemiológicas e desfechos clínicos entre os sexos. Tese (Doutorado) - Centro de Pesquisas Aggeu Magalhães, Fundação Oswaldo Cruz. Recife: 2015.

6. Smeltzer SC, Bare BG, Hinkle JL, Cheever KH. Tratado de enfermagem medico-cirurgica. Rio de Janeiro: Guanabara Koogan, 2005; p. 766-802.

7. Ferreira EF, Assis RC, Faria AKN, Chaves CG, Souza ASD, Zani $\mathrm{HP}$, et al. Perfil epidemiológico e avaliação de fatores de risco para doença cardiovascular em pacientes atendidos em uma clínica escola de fisioterapia. Rev Educ Saúde. 2017;5(2):19-25. https://doi.org/10.29237/2358-9868.2017v5i2.p19-25
8. Scherer C, Stumm EMF, Loro MM, Kirchner RM. O que mudou em minha vida? Considerações de indivíduos que sofreram infarto agudo do miocárdio. Rev Eletr Enf. 2011;13(2): 296-305.

http://dx.doi.org/10.5216/ree.v13i2.8534

9. Trentini M, Paim L, Silva DMG. Pesquisa Convergente assistencial: delineamento provocador de mudanças nas práticas de saúde. Porto Alegre: Moriá, 2014; p.60-114.

10. Pacheco RL, Latorraca COC, Pachito DV, Rieva R. Guidelines para publicação de estudos científicos. Parte 1: como publicar relatos e series de casos. Diagn Tratamento. 2017;22(2):78-82.

11. Minayo MCS. O desafio do conhecimento: pesquisa qualitativa em saúde. São Paulo: Hucitec; 2010. 